\title{
Caso clínico. Lesión obstétrica de plexo braquial C5-C6/C7
}

\author{
Clinical case: $\mathrm{C} 5-\mathrm{C} 6 / \mathrm{C} 7$ obstetric brachial plexus injury
}

René Humberto Barraza Arrambide, ${ }^{\star}$ Jorge Francisco Clifton Correa, ${ }^{\ddagger}$ Leonardo López Almejo, § Ana Fernanda Espinosa de los Monteros Kelley, Raúl Emérico Rodríguez Martínez," Rogelio Josué Solano Pérez, ${ }^{\text {** }}$ Eduardo Pablo Zancollił

* Cirujano Ortopedista, Cirujano de Mano, Cirugía de Nervio Periférico, Staff del Christus Muguerza Hospital Saltillo, Profesor del Diplomado de Cirugía de Plexo Braquial de la Universidad Autónoma de Coahuila (UAdeC), Torreón, Coahuila. Máster en Brachial Plexus International School (BPIS).

‡ Cirujano Plástico, Profesor de Cirugía de Plexo Braquial y Nervio Periférico, Hospital Universitario, UAdeC. Profesor del Máster en Cirugía de Plexo Braquial, BPIS.

$\S$ Cirujano Ortopedista, Cirujano de Mano, Cirugía de Nervio Periférico, Staff del Star Médica Aguascalientes, Profesor del Diplomado de Cirugía de Plexo Braquial de la UAdeC, Torreón, Coahuila. Máster en BPIS.

" Cirujano Ortopedista, Cirujano de Mano, Cirugía de Nervio Periférico, Staff del Hospital Real San José, Guadalajara.

Profesor del Diplomado de Cirugía de Plexo Braquial de la UAdeC, Torreón, Coahuila. Máster en BPIS.

" Cirujano Ortopedista, Cirujano de Mano, Cirugía de Nervio Periférico, Staff del Hospital Puerta de Hierro,

Guadalajara. Diplomado de Cirugía de Plexo Braquial de la UAdeC, Torreón, Coahuila. Máster en BPIS.

${ }^{* *}$ Cirujano Ortopedista, Cirujano de Columna, Cirugía de Nervio Periférico. Staff del Hospital «Dr. Victorio de la Fuente Narváez", Ciudad de México. Diplomado de Cirugía de Plexo Braquial de la UAdeC, Torreón, Coahuila. Máster en BPIS. \# Cirujano Ortopedista, Cirujano de Mano, Cirugía de Nervio Periférico. Staff del Hospital Fundación Favaloro, Buenos Aires, Argentina. Diplomado de Cirugía de Plexo Braquial de la UAdeC, Torreón, Coahuila. Máster en BPIS.

\section{Christus Muguerza Hospital Saltillo. Saltillo, Coahuila, México.}

Correspondencia: Dr. René Humberto Barraza Arrambide. Christus Muguerza Hospital Saltillo, Carretera SaltilloMonterrey Km 4.5, Interior 162, 25210, Saltillo, Coahuila, México. Correo electrónico: cirugiademano.saltillo@gmail.com

\section{RESUMEN}

Las lesiones del plexo braquial obstétricas, pueden presentarse de manera total o parcial, muchas de las lesiones totales evolucionan a la recuperación espontánea, esta recuperación puede mostrar distintas calidades de la función del hombro, codo, muñeca y mano; siendo así, la mayoría de

\section{ABSTRACT}

Obstetric brachial plexus injuries can be present as total or partial, many of the total injuries evolve to spontaneous recovery, this recovery can show different qualities of the function of the shoulder, elbow, wrist and hand, being thus, most of the obstetric brachial plexus injuries will show

Citar como: Barraza ARH, Clifton CJF, López AL, Espinosa de los Monteros KAF, Rodríguez MRE, Solano PRJ y col. Caso clínico. Lesión obstétrica de plexo braquial C5-C6/C7. Ortho-tips. 2020; 16 (4): 212-217. https://dx.doi.org/10.35366/97820 
las lesiones obstétricas del plexo manifestarán cierta recuperación de la función del hombro y flexión del codo, muchas veces incompleta, lo que lleva a la aparición de discinesia del hombro, con la subsecuente displasia del desarrollo glenohumeral, dejando una afectación definitiva de la función del hombro cuando no se logra una reinervación muscular.

Palabras clave: Lesión de plexo braquial, parálisis alta, displasia glenohumeral, lesión perinatal, tratamiento quirúrgico. a certain recovery of the function of the shoulder and flexion of the elbow, often incomplete, which leads to the appearance of shoulder dyskinesia, with the subsequent dysplasia of glenohumeral joint, leaving a definitive impairment of the function of the shoulder, with subsequent glenohumeral developmental dysplasia, leaving a definitive impairment of shoulder function when muscle reinnervation is not achieved.

Keywords: Brachial plexus injury, upper palsy, glenohumeral dysplasia, perinatal injury, surgical treatment.

\section{PRESENTACIÓN DEL CASO}

Se trata de un paciente masculino de dos meses de vida, producto de la quinta gesta, $4.800 \mathrm{~kg}$ al nacer; fue obtenido por parto distócico, cursó con fractura de clavícula izquierda y cursó con hipoxia perinatal que requirió maniobras de reanimación neonatal, permaneciendo en cuidados neonatales por una semana, presentó ictericia neonatal, a decir de los papás, el bebé mostraba datos de parálisis total de la extremidad de recién nacido.

A la exploración física, se observó con capacidad flexora de los dedos y muñeca, con limitación de la extensión de la muñeca y codo, muestra fasciculaciones de los flexores del codo sin capacidad de flexión efectiva, ausencia de capacidad de rotadores externos del hombro, capacidad del deltoides muy disminuida, no logra realizar abducción del hombro. Sin evidencia de signo de Horner. Se indica rehabilitación física, vigilancia y cita en cuatro semanas (Figura 1).

Fue hasta mayo del 2018 que regresan a consulta, ya con 10 meses de edad del paciente, mostrando datos de mejoría de la capacidad de flexores de los dedos, muñeca aún con debilidad para la extensión, no supina, no extiende codo de manera efectiva, flexores del codo con M1. Hombro en rotación interna con capacidad de abducción y rotación externa nulas, muestra flexión anterior a $90^{\circ}$, ayudada por la hiperextensión de la columna para lograr un signo del trompetero. Se le indicó que se le tomara TAC de hombros para determinar el grado de displasia gle-

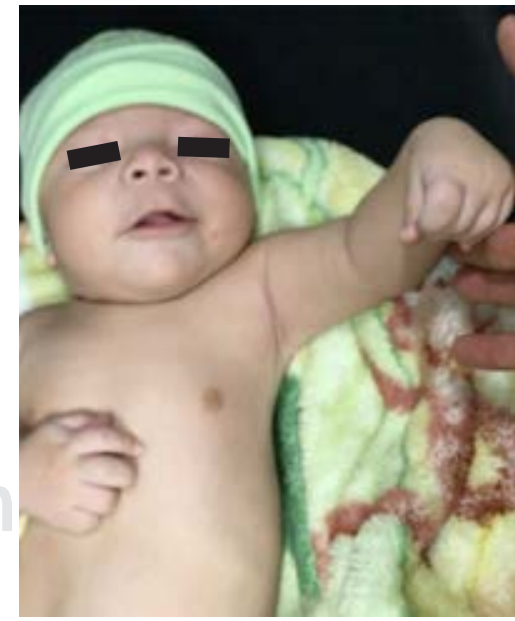

Figura 1: Valoración inicial, brazo flácido con pobre función de la mano. 

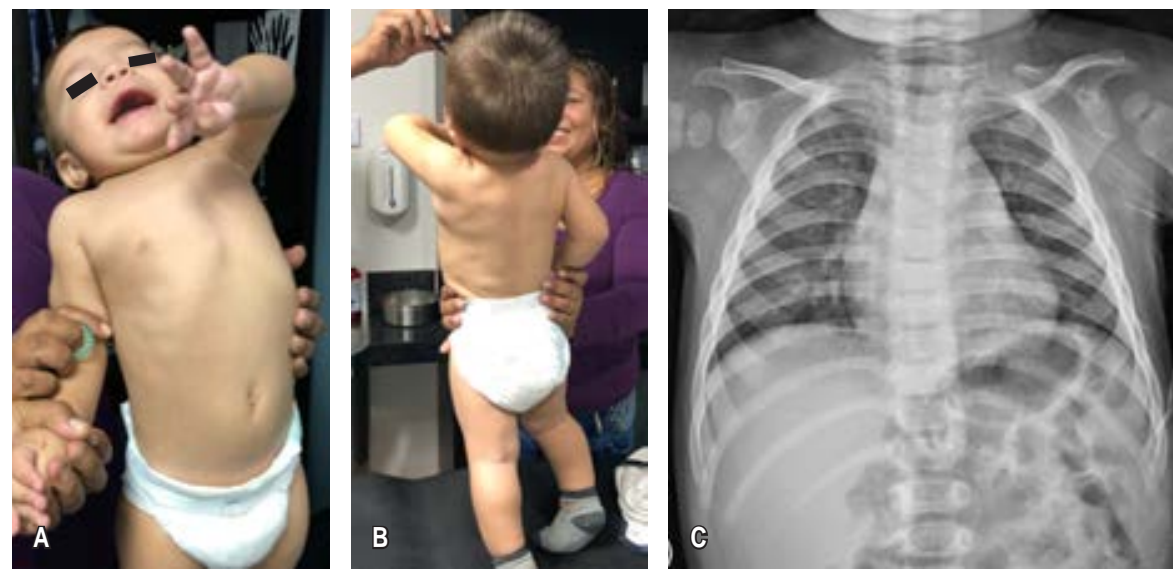

Figura 2: A y B) Capacidades de hombro preoperatorias, signo del trompetero. C) $\mathrm{Rx}$ demostrativa de hipoplasia de núcleos de crecimiento de húmero proximal izquierdo.

nohumeral, y se le propone tratamiento quirúrgico, el cual se programará una vez que tenga los estudios de imagen.

El caso de nueva cuenta acude a cita hasta mediados de julio del 2018, a causa de que había permanecido hospitalizado por tres semanas por infección gastrointestinal y ya recuperado. Se observa con mejoría de los rangos de movimiento de los extensores de muñeca y dedos y tríceps, bíceps débil, función de hombro sin cambios, lo que lo ubicaba en un tipo II de la clasificación de Mallet. No practicaron TAC, pero sí realizaron rayos $\mathrm{x}(\mathrm{Rx})$ del tórax, lo cual muestra datos de hipoplasia de núcleos de crecimiento de la cabeza humeral izquierda en comparación con la derecha, que sugiere glenohumeral displasia mínima, por lo que se programa cirugía de exploración quirúrgica de plexo braquial, valorar reconstrucción de TPS, transferencias de Obserlin y de XI a supraespinoso y liberación anterior del hombro (Figura 2).

El día 14 de septiembre fue operado y se le realizó exploración de plexo braquial izquierdo, encontramos un gran neuroma en C5-C6/C7 hasta las divisiones de TPS y TPM, se hizo resección parcial del neuroma, observando que conduce de manera deficiente para musculatura de hombro a la estimulación pero de manera más efectiva para la flexión de la muñeca y codo, se hizo reconstrucción de C5 a DPTPS, C6 a DATPS con injerto de nervio sural de pierna izquierda, y transferencia de Xla a nervio supraescapular y transferencia de nervio mediano (rama de FCR) a nervio musculocutáneo, rama para bíceps.

Además se le practicó liberación anterior de hombro, con liberación de ligamento coracohumeral y coracoidectomía. Al término del procedimiento se le colocó un aparato toracobraquial de fibra de vidrio con abducción, el cual fue retirado a las seis semanas, para retomar así terapias de rehabilitación física (Figura 3). 

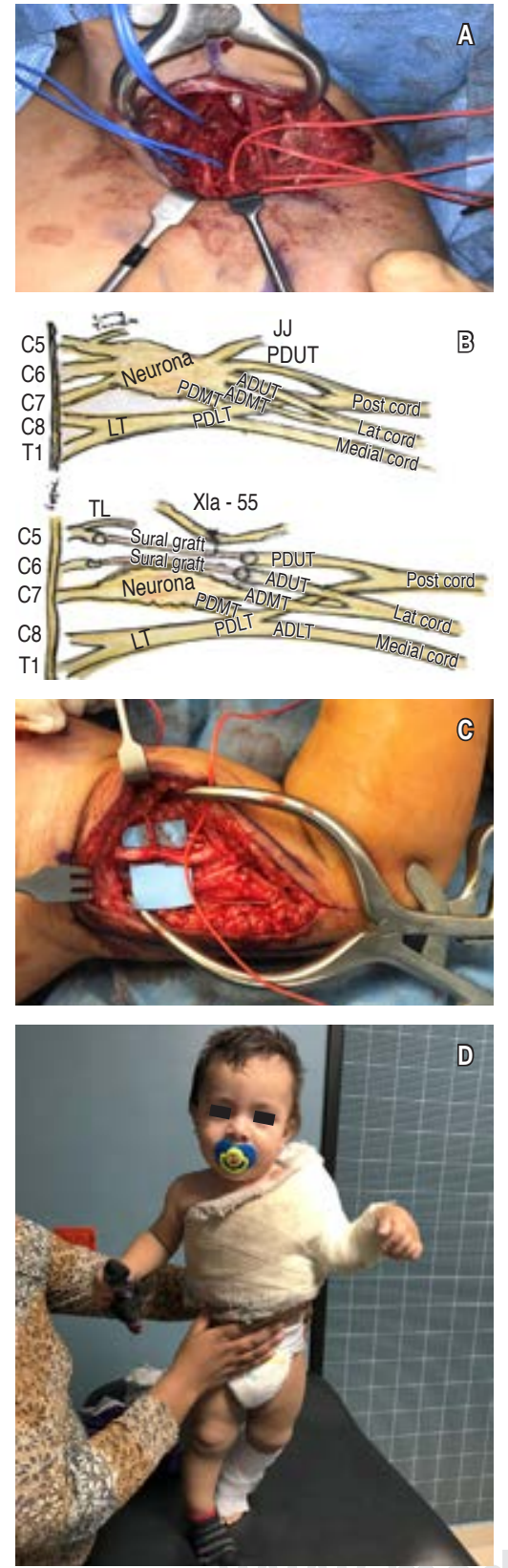

Figura 3: Fotografías transoperatorias. A) Neuroma del TPS y TPM. B) Esquema de hallazgos quirúrgicos y estrategia quirúrgica realizada. C) Trasferencia de Oberlin (mediano-musculocutáneo). D) Aparato de inmovilización toracobraquial.
Seguido de la inmovilización, se llevó a cabo protocolo de rehabilitación física donde se observa que el sujeto logra capacidades de flexión y extensión activa del codo y logra actividades de rotación externa activa de hombro, con abducción y flexión anterior por encima de los $90^{\circ}$ que lo coloca en un score IV de la clasificación de Mallet (Figura 4).

\section{DISCUSIÓN DEL CASO}

Este es un típico caso de lesión del plexo braquial perinatal en el cual el paciente resulta con una lesión total y a lo largo de la evolución, muestra datos de recuperación espontánea, lo cual conlleva que tanto los familiares como los equipos de rehabilitación consideren que el paciente se recuperará y por lo tanto descartan la necesidad de una cirugía.

En una parálisis total, el observar algo de recuperación de función de la mano es alentador, aunque esto no garantiza la recuperación de las funciones del codo y hombro, y siendo así, no siempre estas funciones se logran normales. Este fenómeno se puede observar en lesiones donde se presenta elongación, ruptura del TPS y elongación del TPI.

Regularmente en estos casos la elongación del TPI se resuelve de manera espontánea y la ruptura del TPS, al intentar curar, genera un neuroma que puede conducir algo de función, la cual suele ser ineficiente o facilitar el desarrollo de cocontracturas en músculos del hombro y de los flexoextensores del codo. ${ }^{1,2}$

Esto se pone en evidencia cuando observamos un hombro recuperado pero que no logra la rotación externa pasiva a expensas de la contractura de los rotadores internos (subescapularis), así como la presencia de signo de Putti, que representa la contractura de la 
musculatura del hombro y pone en relieve el movimiento toracoescapular del hombro más que el movimiento glenohumeral. ${ }^{1-3}$

La falta de flexión del codo obedece a una falla en la reinervación de la musculatura del bíceps y del braquial, y lo que se alcanza a observar de flexión del codo regularmente proviene de un fenómeno de Steindler. La flexión del codo es compensada por el FCR, esto lo podemos identificar porque la flexión del codo se les facilita con el antebrazo en pronación y al supinarlo pierden la capacidad de flexionar, aunque este tipo de observaciones son sumamente difíciles de hacer en un niño pequeño, por lo que se debe palpar intencionalmente en busca del movimien-

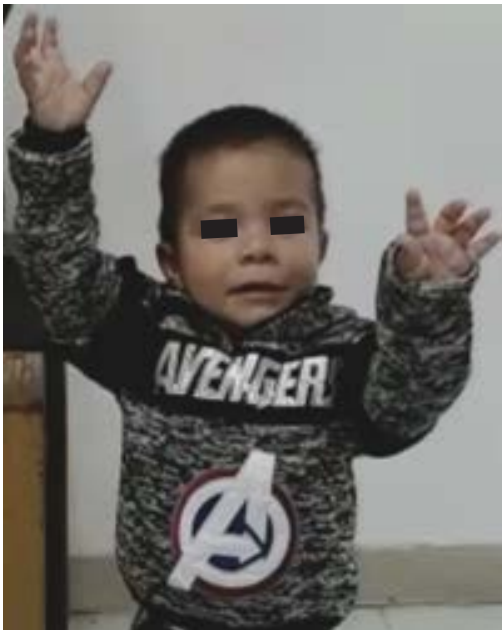

Figura 4: Resultado postoperatorio a los dos años de vida. to del tendón del bíceps. ${ }^{1}$

Cuando hicimos la exploración quirúrgica de este caso, advertimos la formación de un gran neuroma del TPS y TPM, una vez identificadas las raíces C5 y C6 viables, se realizó reconstrucción con injerto nervioso de sural hacia las divisiones anterior y posterior de TPS, con esto se busca recuperar la inervación de los músculos de hombro y de los flexoextensores del codo, así como para recuperar la inervación sensitiva de dichas regiones, ${ }^{4}$ y nos apoyamos en el uso de transferencias nerviosas intra y extraplexales para potenciar la motricidad.

Aunado a esto, la liberación anterior del hombro y valorar hacer la coronoidectomía incrementan la oportunidad de que la musculatura reinervada no tenga limitantes osteoarticulares para funcionar lo mejor posible.

Los factores biológicos inherentes a la lesión definitivamente dictan el pronóstico de ésta; sin embargo, en estos casos el pronóstico también se ve afectado por las condiciones sociales, como la atención temprana, indecisión de los familiares y el equipo médico en indicar y realizar una cirugía, lo cual puede afectar de manera definitiva el curso de la enfermedad y a veces facilitar la instalación de secuelas que podrían ser evitadas por un actuar oportuno. ${ }^{2}$

\section{CONCLUSIÓN}

El conocimiento de la historia natural de estas lesiones, la correcta toma de decisiones y el timing adecuado al momento de hacer una cirugía y realizar de manera adecuada los procedimientos mejoran las oportunidades de recuperación de los casos con lesiones del plexo braquial perinatal.

Aunado a esto, una estrecha comunicación y educación continua a padres del bebé, equipo médico, rehabilitadores, trabajadores sociales y psicólogos 
puede brindar la diferencia en la evolución del tratamiento de las lesiones del plexo braquial en bebés.

\section{BIBLIOGRAFÍA}

1. Borrero JL. Obstetrical brachial plexus palsy. Lima: Althaus; 2007.

2. Martin E, Senders JT, DiRisio AC, Smith TR, Broekman MLD. Timing of surgery in traumatic brachial plexus injury: a systematic review. J Neurosurg. 2018: 1-13.

3. Al-Qattan MM. Obstetric brachial plexus injuries. J Am Soc Surg Hand. 2003; 1 (3): 41-54.

4. Laurent JP, Lee R, Shenaq S, Parke JT, Solis IS, Kowalik L. Neurosurgical correction of upper brachial plexus birth injuries. J Neurosurg. 1993; 79 (2): 197-203. 